\title{
Launch of Emergent Scientist: hands on peer-review publication for science students
}

Daniel Suchet

Emergent Scientist Editor-in-Chief

emergent.scientist@edpsciences.org

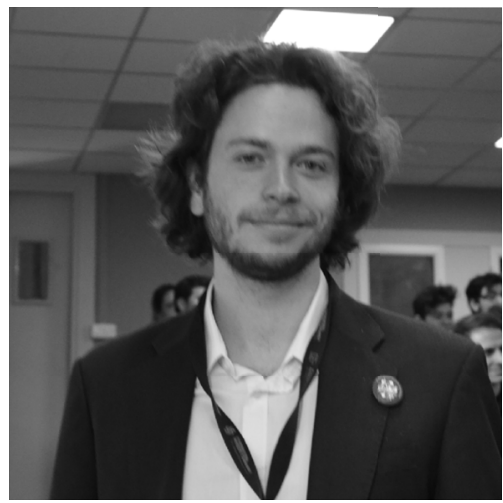

Peer review publishing is at the very heart of science. It is this impartial cross-validation by independent experts that makes research reliable and guarantees a certain level of scientific rigour. To understand how science works, and how societies can rely on it, it is therefore crucial not only to practice the scientific approach in study and class projects, but also to experience the publication process itself. We thus believe an introduction to the peer-review process is a valuable asset for science students, regardless of whether their curricula lead them to do research or not.

This pedagogical demand, however, conflicts with the expectations of most scientific journals: research articles explore the frontiers of knowledge, while studying is precisely about reaching these frontiers. How are you supposed to publish something new when you do not yet know what is already known? Emergent Scientist (Em. Sci.) short-circuits this issue by offering young scientists an opportunity to discover peer-reviewed publishing.

Emergent Scientist is an open access journal published by EDP Sciences, and supported by the French Physics Society and the French Academy of Science. Just like any other journal, we receive manuscripts, ask referees to review them, supervise their revision by the authors, and publish them once they reach a satisfactory level of scientific quality. However, our assessments are quite different from those of mainstream publications: we do not expect authors to exhibit groundbreaking results, but to show exemplary scientific rigor and clarity in the way they tackle a problem and report their findings. By doing so, we also aim at building an open-access database of scientific publications that will be accessible to students and constitute examples of sound investigation methods.

Obviously, Emergent Scientist is not constrained by the same standards of innovation as other journals. We want to use this flexibility to emphasize critical steps of research that are too often underreported. For instance, all articles will feature a "dead end" section, where unsuccessful leads are outlined so that the reader will acknowledge how failure is inherent to research - and hopefully avoid repeating the same mistake over and over again. We also allow for the publication of several articles on the same topic, as we consider it insightful to see the diversity of methods employed in tackling a single issue. Over time, Emergent Scientist also aims at granting young scientists editorial responsibilities, and involving students at every step of the publication process.

At the present time, Emergent Scientist is a project managed by a handful of physicists and a professional publisher. Emergent Scientist is a bet. We bet that colleagues from other fields will join us and broaden our scope. We bet that students will be interested to turn their research projects into proper scientific articles. We bet that such articles will be valuable both for their authors and for readers who have little access to professional journals. We bet that universities and other scientific institutions will join us and encourage their students and teachers to seize the opportunities offered by Emergent Scientist.

Are you game?... 